\title{
Confinement Of Pure Ion Plasma In A Cylindrical Current Sheet
}

\author{
Stephen F. Paul, Edward H. Chao, Ronald C. Davidson, \\ Cynthia K. Phillips \\ Plasma Physics Laboratory \\ Princeton University, Princeton, New Jersey 08543
}

\begin{abstract}
A novel method for containing a pure ion plasma at thermonuclear densities and temperatures has been modeled. The method combines the confinement properties of a Penning-Malmberg trap and some aspects of the magnetic field geometry of a pulsed theta-pinch. A conventional Penning trap can confine a uniform-density plasma of about $5 \times 10^{11} \mathrm{~cm}^{-3}$ with a 30 -Tesla magnetic field. However, if the axial field is ramped, a much higher local ion density can be obtained. Starting with a $10^{7} \mathrm{~cm}^{-3}$ trapped deuterium plasma in a conventional Penning-Malmberg trap at the Brillouin limit ( $\mathrm{B}=0.6$ Tesla), the field is ramped to 30 Tesla. Because the plasma is comprised of particles of only one sign of charge, transport losses are very low, i.e., the conductivity is high. As a result, the ramped field does not penetrate the plasma and a diamagnetic surface current is generated, with the ions being accelerated to relativistic velocities. To counteract the inward $j \times B$ forces from this induced current, additional ions are injected into the plasma along the axis to increase the density (and mutual electrostatic repulsion) of the target plasma. In the absence of the higher magnetic field in the center, the injected ions drift outward until a balance is established between the outward driving forces (centrifugal, electrostatic, pressure gradient) and the inward $j \times B$ force. An equilibrium calculation using a relativistic, 1-D, cold-fluid model shows that a plasma can be trapped in a hollow, 49-cm diameter, $0.2-\mathrm{cm}$ thick cylinder with a density exceeding $4 \times 10^{14} \mathrm{~cm}^{-3}$.
\end{abstract}

\section{INTRODUCTION}

This paper presents a novel method for achieving a well-known goal: the confinement of non-neutral ion plasmas that are adequately dense for controlled thermonuclear fusion applications. This approach is a subset of a wider class of experiments known as Inertial Electrostatic Confinement experiments [1], but the question addressed here is quite specific: can the density of particles in a non-neutral plasma [2] be increased significantly beyond the density associated with the Brillouin limit?

Conventional magnetic fusion devices contain quasi-neutral plasmas in a toroidal or linear geometry, but generally the confinement is far worse than that predicted 
classically. A common device used to confine a non-neutral plasma is the cylindrical Malmberg-Penning trap [3]. Though at first glance non-neutral plasmas would not appear to be good candidates for long confinement time applications (because of the strong, outward electrostatic forces associated with the space-charge potential), Malmberg-Penning traps have exhibited superb confinement capability. This is because the conservation of rotational angular momentum requires the mean square radius of a one-component plasma to remain constant in time. One-component plasmas also have two other benefits from a practical standpoint for fusion devices: (1) the absence of radiative losses because electrons are not present and (2) reduced thermal wall loading because of the sharp radial fall-off in the density profile (typically on the order of several Debye lengths). This superior confinement capability has not been generally realized in controlled fusion devices, however, because conventional Malmberg-Penning traps can confine uniform-density plasmas of only about $5 \times 10^{11} \mathrm{~cm}^{-3}$, even with magnetic fields strengths in the tens of Tesla. This is expressed as the Brillouin limit,

$$
2 \frac{\omega_{p}^{2}}{\omega_{c}^{2}}<1 \quad \text { or } \quad n<\frac{B^{2}}{8 \pi m c^{2}},
$$

where $\omega_{p}$ is the plasma frequency, $\sqrt{4 \pi n e^{2} / m}$, and $\omega_{c}$ is the cyclotron frequency, $e B / m c$. The plasma column is rotating at $\omega_{R}$, which is equal to $\omega_{c} / 2$ at the Brillouin limit. Equation (1) shows that higher density may be achieved only if the square of the magnetic field is increased proportionally. Even at such high field strengths, the Lawson criterion is satisfied only if the confinement time is nearly 1 hour.

Though most often viewed as a density limit, the Brillouin limit is really a statement of the fact that the inward $j \times B$ force must balance the outward driving forces (centrifugal, electrostatic, pressure gradient). For plasmas that do not have a uniform density, force balance can be achieved even though local values of $n$ far exceed the limit expressed in Eq. (1). In this paper, the simple geometry of the Penning trap is retained; the confinement device proposed provides radial confinement through an axial magnetic field, and longitudinal confinement electrostatically by end cylindrical electrodes kept at a potential higher than the plasma's space charge potential. The difference is that, unlike in a conventional Penning trap, the axial magnetic field is highly non-uniform, similar to the implosion stage of a theta-pinch. In addition, the velocity shear in the device is very large and the density profile is not monotonically decreasing. These two factors could have serious consequences where the stability of the configuration is concerned, but these are not addressed here.

\section{METHODOLOGY}

A highly non-uniform field is created by initially producing a conventional, lowdensity Malmberg-Penning trap plasma, followed by ramping the axial field. For example, a uniform density, $10^{7} \mathrm{~cm}^{-3}$ deuterium plasma might be generated at the 
Brillouin limit (for $B=0.6$ Tesla), after which the field is slowly ramped to as high a value as is practical (30 Tesla). Because the plasma is comprised of particles of only one sign of charge, transport losses are very low, i.e., the conductivity is high. As a result, the ramped field does not penetrate the plasma as long as the ramping time is short compared to the magnetic diffusion time. A diamagnetic surface current is generated, and, in this example, the ions are accelerated to relativistic velocities, with the magnetic field outside the plasma 50 times stronger than inside. To counteract the inward $j \times B$ forces, ions are injected along the machine axis to increase the density (and mutual electrostatic repulsion) of the target plasma. With only the weak magnetic field present within the bulk of the plasma, the ion density inside the plasma column greatly exceeds the Brillouin limit and the ions drift outward. Ultimately, a balance is established between the outward driving forces (centrifugal, electrostatic, pressure gradient) and the inward $j \times B$ force, and the plasma is trapped in a thin current sheet between its own outward electrostatic repulsive forces and the confining magnetic field. Note that the field is ramped only to generate a non-uniform $B$, which is in contrast to a theta-pinch approach, in which the coil is pulsed to compress and heat the plasma.

\section{EQUILIBRIUM MODELING}

To determine the characteristics of such an equilibrium, a relativistic 1-D coldfluid model was used to model the system. Starting with the radial force balance equation for a cold macroscopic fluid [3],

$$
-\frac{\gamma_{\theta} m \beta_{\theta}^{2} c^{2}}{r}=e\left[E_{r}+\beta_{\theta} B_{z}\right],
$$

where

$$
\beta_{\theta}(r)=\frac{V_{\theta}(r)}{c},
$$

operating on it with $1 / r \partial / \partial r[r \ldots]$, and substituting in Ampere's law and Gauss' law leads to an equation for the derivative of $\beta_{\theta}(r)$ as a function of $\beta_{\theta}(r), B_{z}$, and $\partial B_{z} / \partial r$ :

$$
\frac{\partial \beta_{\theta}}{\partial r}=\frac{1}{\gamma_{\theta}^{2} \beta_{\theta}} \frac{\frac{1}{B_{z}} \frac{\partial B_{z}}{\partial r}-\frac{\gamma_{\theta}^{2} \beta_{\theta}^{2}}{r}}{1+\gamma_{\theta} \beta_{\theta} \frac{m c^{2}}{e B_{z} r}\left(\gamma_{\theta}^{2} \beta_{\theta}^{2}+2\right)},
$$

where

$$
\gamma_{\theta}(r)=\frac{1}{\sqrt{1-\beta_{\theta}^{2}}} .
$$


This first order ODE is solved using Mathematica with the boundary condition $\beta_{\theta}\left(r=0^{+}\right)=0^{+} . B_{z}$ is specified as nearly a step function located at $r_{\circ}$ with penetration depth $d_{\circ}$ :

$$
B_{z}(r)=\frac{B_{\circ}}{2}\left(1+\operatorname{Tanh}\left[\frac{\left(r+r_{\circ}\right)\left(r-r_{\circ}\right)}{2 d_{\circ}^{2}}\right]\right) .
$$

$r_{\circ}$ and $d_{\circ}$ are chosen such that fusion power is maximized by increasing the ion density and plasma volume to the greatest extent possible. To that end, 30 Tesla is chosen for the ramped field to illustrate the potential of this approach. A simplified relativistic force balance estimate shows that the plasma can be trapped in a hollow, 50-cm diameter, $0.2-\mathrm{cm}$ thick cylinder, whose density exceeds $4 \times 10^{14} \mathrm{~cm}^{-3}$. The drawback, of course, is the very small thickness of the current sheet, resulting in a low plasma volume. For this simulation, the radius is maximized so that the resulting plasma volume is large, constrained only by the requirement that the local velocity not exceed the speed of light. This estimate indicates that $2 \mathrm{MW}$ of fusion power could be produced in a 100-m long, 1-m diameter reactor. At $72 \mathrm{~m}^{3}$, the device would be only $4 \%$ of the volume of ITER, while a device with the same volume as ITER would produce $55 \mathrm{MW}$. In the fluid simulation, the thickness of the shell is set at 2 Larmor radii, i.e, the ramped B-field is assumed not to penetrate the highly conducting ion plasma at all. The other parameters are: $B_{\circ z}=300 \mathrm{kG}$, $r_{\circ}=49.0$, and $d_{\circ}=1.85 \mathrm{~cm}$. The magnetic field in the region of the field transition given in Eq. (4) is shown in Fig. 1.

Given the magnetic field profile, the velocity profile is uniquely determined by solving Eq. (3). In this case, the velocity is relativistic with $\beta_{\theta}$ about 0.85 . The rotation frequency profile, $\omega(r)=c \beta_{\theta}(r) / r$ is determined from the velocity and is shown in Fig. 2. Given the velocity profile, the density profile can be readily calculated from Ampere's law:

$$
n(r)=\frac{1}{4 \pi e \beta_{\theta}(r)} \frac{\partial B_{z}}{\partial r} .
$$

The plasma forms a thin cylindrical shell in the region of the magnetic field transition, which is shown in Fig. 3. The current density is easily calculated from its definition:

$$
j(r)=n(r) e \beta_{\theta}(r) c
$$

The very high current density (several MA $/ \mathrm{cm}^{2}$, shown in Fig. 4.), confines the plasma through the inward radial $j \times B$ force. Given the density profile, the electric field is calculated from Gauss' law:

$$
E(r)=300 \times 10^{6} \cdot 4 \pi e \frac{1}{r} \int n\left(r^{\prime}\right) r^{\prime} d r^{\prime}
$$

The self electric field generated at the surface of the plasma as calculated from Eq. (7). is large (100's of MV/cm). However, there is little charge or field inside the plasma shell, as shown in Figs. 3 and 5. 


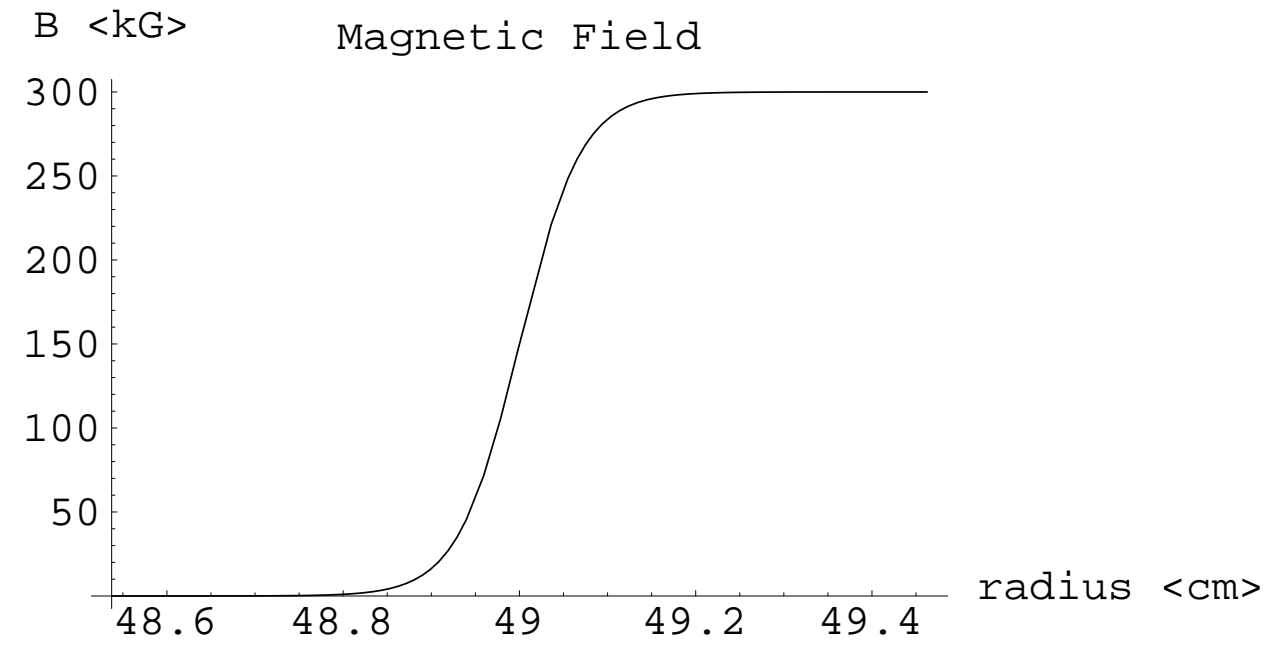

FIGURE 1. The magnetic field exhibits a strong gradient at the surface of the initial, low-density plasma.

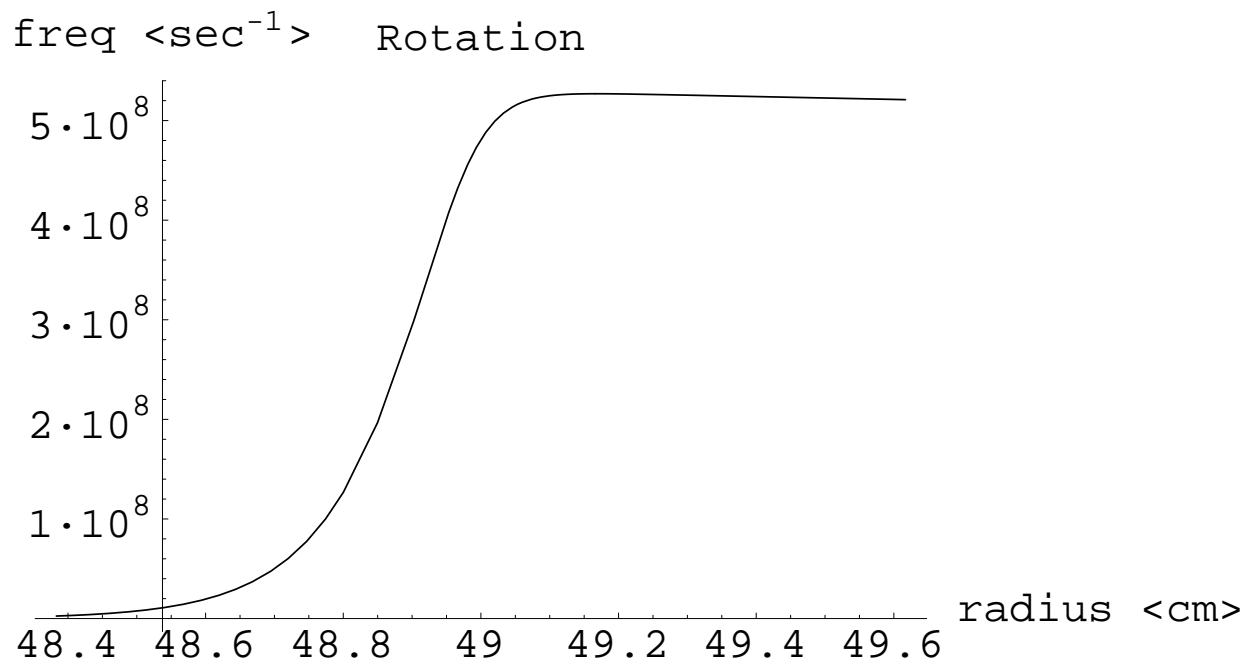

FIGURE 2. The rotation frequency profile in the region of the plasma shell. 


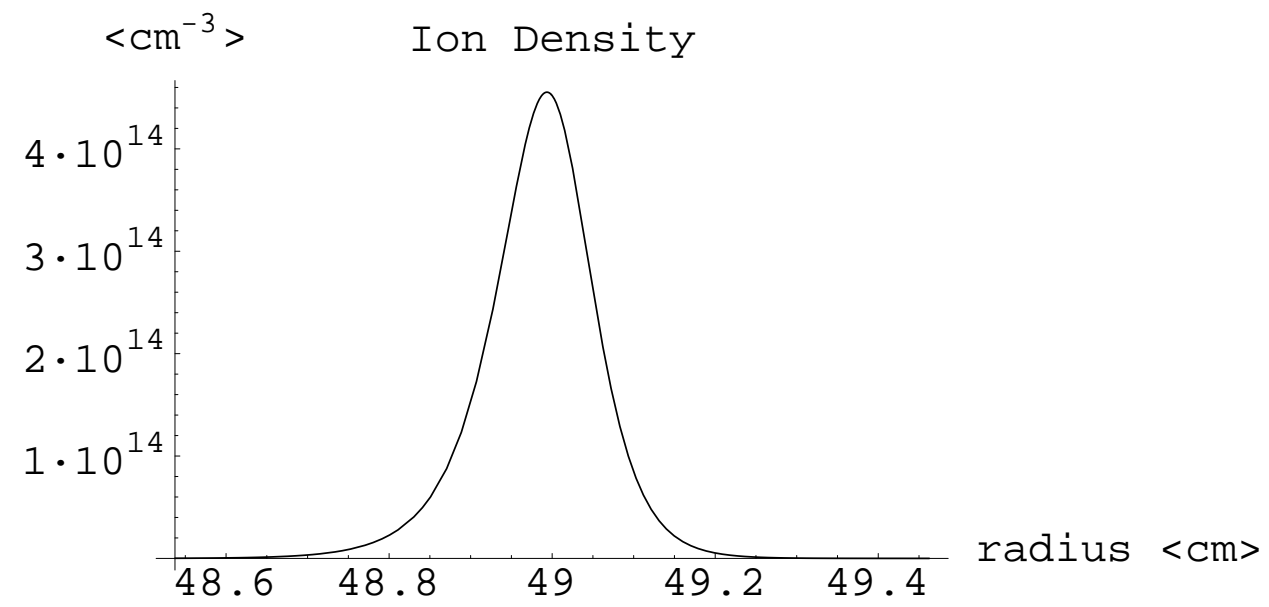

FIGURE 3. The density is a thin sheet of current located at the magnetic field gradient.

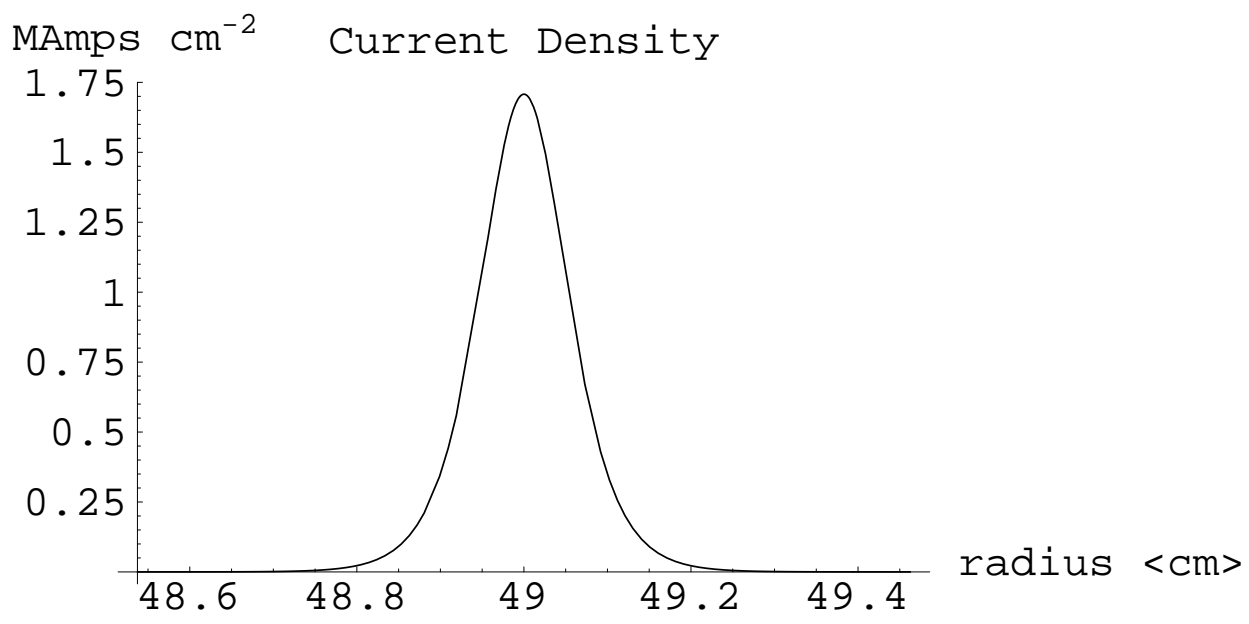

FIGURE 4. A very high current density provides the confining forces. 


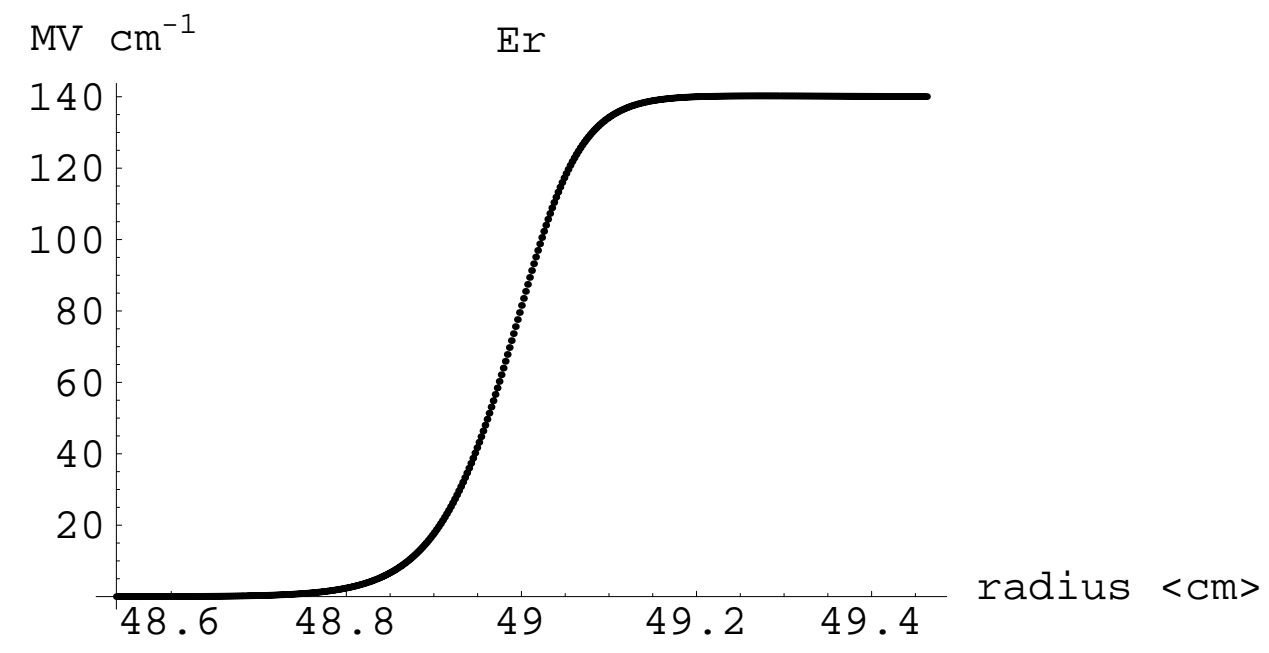

FIGURE 5. The electric field in the region of the transition.

\section{INCLUSION OF FINITE TEMPERATURE}

To determine how the inclusion of finite temperature affects the characteristics of the cold fluid equilibrium, a relativistic pressure gradient term, $1 / \mathrm{n} \nabla P$, is added to the 1-D, cold-fluid model. A simplified model of pressure, with varying density but isotropic temperature is included in Eq. (2) by adding $T / n(r) \partial n(r) / \partial r$ to the right hand side. Similarly, operating on this equation with $1 / r \partial / \partial r[r \ldots]$ and substituting Ampere's law and Gauss' law as with Eq. (2) produces a second-order differential equation giving $\partial B_{z}^{2} / \partial r^{2}$ as a function of $\beta_{\theta}(r), \partial \beta_{\theta}(r) / \partial r, B_{z}, \partial B_{z} / \partial r$, $\partial B_{z}^{2} / \partial r^{2}$, and $\partial^{3} B_{z} / \partial r^{3}:$

$$
\begin{aligned}
\frac{\partial^{2} \beta_{\theta}}{\partial r^{2}} & =\frac{1}{\beta_{\theta}}\left(\frac{\partial \beta_{\theta}}{\partial r}\right)^{2}+\frac{e \beta_{\theta}}{T}\left\{\frac{1}{\beta_{\theta} \gamma_{\theta}^{2}} \frac{\partial B_{z}}{\partial r}-\frac{\beta_{\theta} B_{z}}{r}\right. \\
& +\frac{T}{e}\left[\frac{\left(\frac{\partial^{2} B_{z}}{\partial r^{2}}\right)^{2}-\frac{\partial B_{z}}{\partial r} \frac{\partial^{3} B_{z}}{\partial r^{3}}}{\left(\frac{\partial B_{z}}{\partial r}\right)^{2}}+\frac{\frac{\partial^{2} B_{z}}{\partial r^{2}}}{r \frac{\partial B_{z}}{\partial r}}\right] \\
& \left.-\left[B_{z}+\frac{m c^{2}}{e r}\left(\gamma_{\theta}^{3} \beta_{\theta}^{3}+2 \gamma_{\theta} \beta_{\theta}+\frac{T}{\beta_{\theta} m c^{2}}\right)\right] \frac{\partial \beta_{\theta}}{\partial r}\right\} .
\end{aligned}
$$


At the temperatures considered here $(\mathrm{T}=80 \mathrm{keV})$, the energy associated with the pressure gradient is only about $0.3 \%$ of the rotational energy of the ions or the electrostatic energy associated with the space charge. Consequently, the qualitative results of the simulation are nearly identical to the cold fluid case. The peak ion density confined with the same field strength is identical to the cold fluid case, as shown in Fig. 3 but there is a small extension in the distribution of ion density towards the center of the configuration, as seen in Fig. 6. The rotation frequency profile is steeper at $48.7 \mathrm{~cm}$ than in the cold fluid case, and tapers off more sharply at $r>49.0 \mathrm{~cm}$. The current density and the electric field remain identical to the cold fluid case.

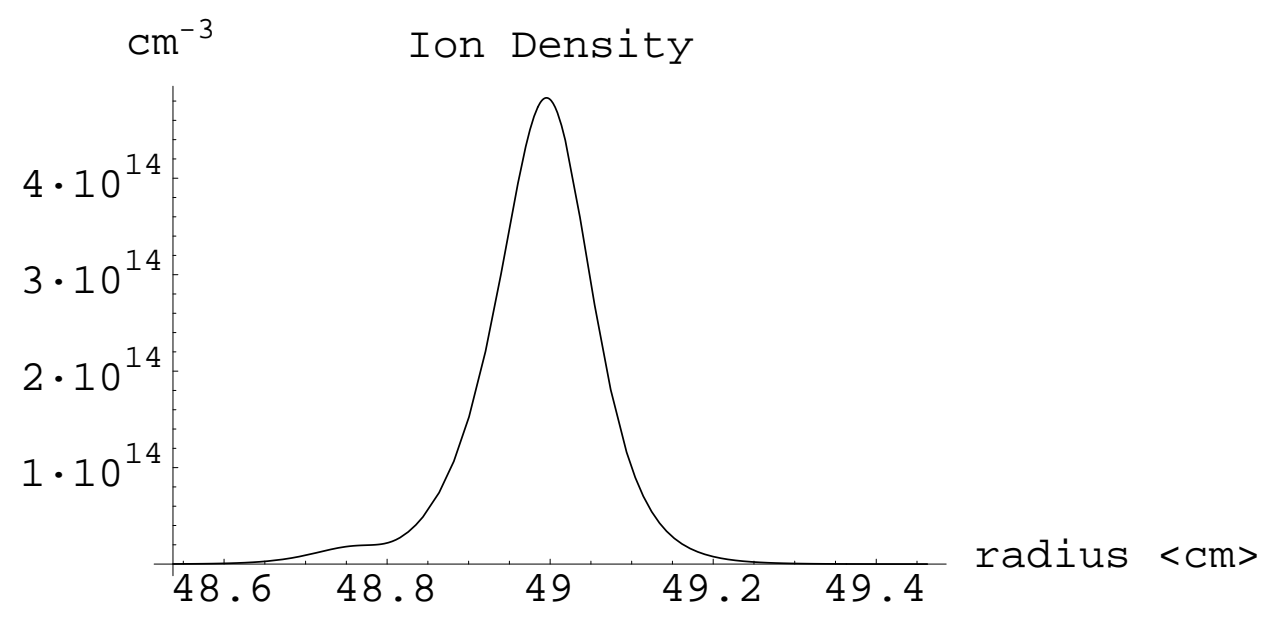

FIGURE 6. The ion density including finite temperature effects.

\section{A TEST CASE: PROPOSED EXPERIMENT FOR THE EDG}

This concept can be easily tested in existing Malmberg-Penning traps. The cold fluid equations are solved for electrons as the plasma species and for parameters suited to the EDG device at Princeton $[4,5]$. For a very modest field that is ramped up to .01 Tesla and a plasma diameter of $1.8 \mathrm{~cm}$, the peak density is $<8.6 \times$ $10^{11} / \mathrm{cm}^{3}$. The rotation frequency is much higher than in the pure ion case because of the much lower mass of the electrons. Peak rotation frequencies of $8 \mathrm{GHz}$ are found as shown in Fig. 7. This rotation corresponds to a $\beta_{\theta}$ of about 0.45 . The peak current density is about $1.6 \mathrm{kA} / \mathrm{cm}^{2}$. The main technical difficulty in performing an experiment in EDG is ensuring that the walls of the cylinder do not break down 
because of field emission. The electric fields, even for this low density, peak at about $90 \mathrm{kV} / \mathrm{cm}$, as shown in Fig. 8 .

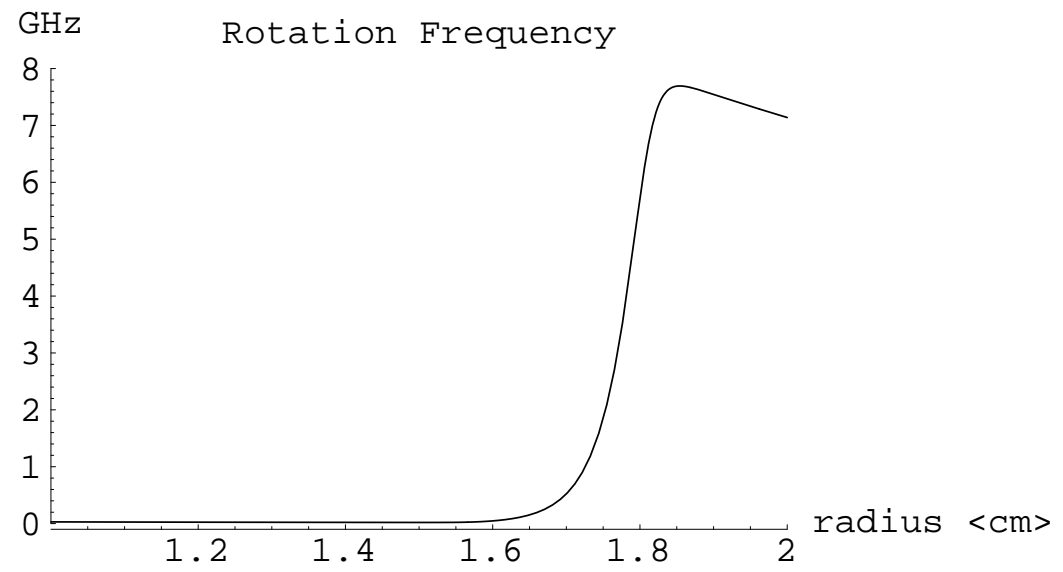

FIGURE 7. The rotation frequencies in EDG.

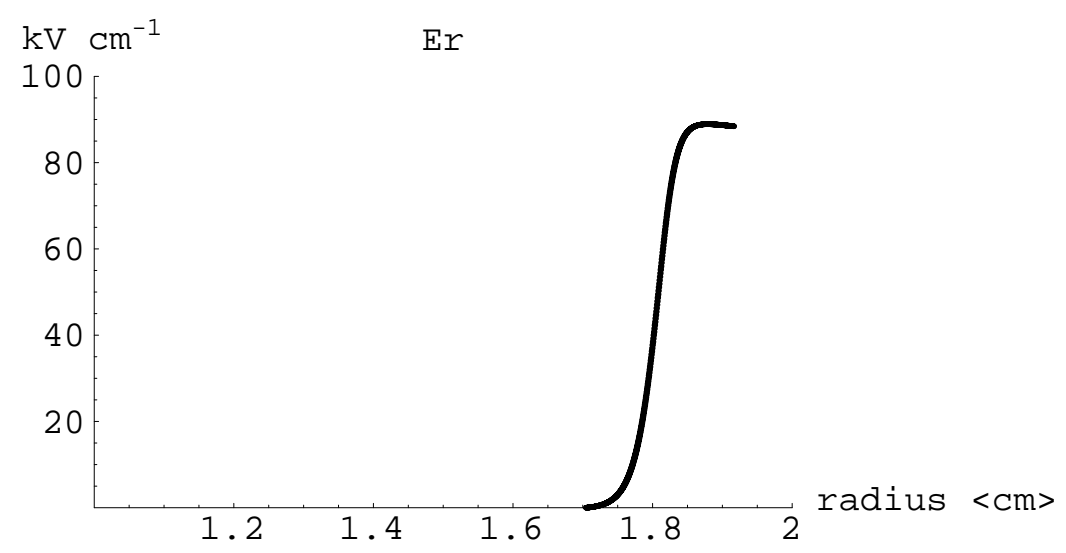

FIGURE 8. The electric field in EDG.

\section{ACKNOWLEDGMENTS}

This research was supported by the Office of Naval Research. The authors gratefully acknowledge Kyle Morrison for useful discussions and help in preparing this manuscript. 


\section{REFERENCES}

1. Barnes, D.C., Mitchell, T.B., Schauer, M.M., Phys. Plasmas 4, 1745 (1997).

2. Barnes, D.C., Nebel, R.A., Turner, L., Tiouririne, T.N., Plasma Phys. Controlled Fusion 35, 929, (1993).

3. Davidson, R.C., Physics of Nonneutral Plasmas, Redwood City, California: AddisonWesley Publishing Company, 1990.

4. Chao, E.H., Davidson, R.C., and Paul, S.F., J. Vac. Sci. Tech. A17, 2050 (1999).

5. Chao, E.H., Paul, S.F., and Davidson, R.C., J. Vac. Sci. Tech. A17, 2034 (1999). 